\title{
Utility of Hematology Histograms
}

\section{Daksha Prabhat, Tejaswini Waghmare and Tasneem Rangwala}

\author{
Department of Pathology, Seth GS Medical College and KEM Hospital, Mumbai, India
}

\begin{abstract}
Background: Over the past five decades, hematology analyzers have evolved from semi-automated to fully automated ones.A histogram is a graphic representation of a collection of data based on cell size and/or cell number depicting variations in the process.

Objective: To study hematology histograms in relation with major blood cell components and in cases of acute febrile illness.

Methods: It was a cross sectional observational study conducted over 200 consecutively collected samples from adults. Histograms were obtained from 3-part automated hematology analyzer,evaluated in relation to the RBCs, WBCs and platelets with their peripheral blood smear (PBS) picture and with the cases with diagnosis of acute febrile illness.

Results: In the present study, maximum number of samples were from 15-25 years of age group with a male preponderance. Out of 200 histograms, 102 had combined abnormality of the all 3 blood cell types in varied combinations.Neutrophilia, microcytosis and thrombocytopenia were the most common findings in the respective individual blood cell histograms. Maximum sensitivity of WBC histogram was for neutrophilia (81.08\%), that of RBC histogram was for microcytosis $(81.3 \%)$ while that of platelet histogram was for thrombocytopenia (97.5\%). In cases of acute febrile illness, dengue was the most common diagnosis with thrombocytopenia being most common histogram finding.
\end{abstract}

Conclusion: Histograms provided by the automated hematology analyzers are of great diagnostic and morphologic importance. Histograms obtained from automated analyzers should be complementary to hematological parameters and peripheral blood smear examination.

Keywords: Automated Hematology analyzers, Hematology Histograms, Sensitivity Of Histograms, Acute Febrile Illness.

\section{Introduction}

Over the past five decades, hematology analyzers have evolved from semi-automated to fully automated ones. They provide rapid and inexpensive cell counting technique. Many additional parameters have become available now. Currently, automated cell counters are the backbone of hematology laboratories.

Blood cells are poor conductors of electricity. When a stream of cells in a conducting medium flows through a small aperture across which an electric current is applied, there is a measurable increase in the electrical impedance across the aperture as each cell passes through, this increase being proportional to the volume of conducting material displaced. The change in impedance is therefore proportional to the cell volume. Cells can thus be both counted and sized from the electrical impulses that they generate. This is the principle of impedance counting, which was devised and developed by Wallace Coulter in the 1940s and 1950s and which ushered in the modern era of automated blood cell counting. ${ }^{[1]}$

However, even in the wake of much technological advancement, the attention to numerical data with regards to the interpretation of test results has not changed. In most laboratory setups, traditional emphasis has been placed on verifying the automated data, an exercise that has outlived its importance. ${ }^{[1]}$

A histogram, also known as a frequency of distribution curve, is a graphic representation of a collection of data based on cell size and/or cell number depicting variations in the process. As graphical data presentation is easier to understand, the histogram is a very powerful tool in morphological analysis of red blood cells (RBCs), white blood cells (WBCs) and platelets. It allows the users to intuitively see the visual comparison of the centre and spread of data and data containing 2 or more variables. ${ }^{[2]}$

With this backdrop, the current study was planned to study hematology histograms in relation with major blood cell components and cases of acute febrile illness.

\section{Materials and Methods}

Ethics: Prior approval was taken from Institutional Ethics Committee before commencing the study. A written informed consent was taken from patients.

Methodology: It was a cross sectional observational study conducted over 200 consecutively collected samples in the Department of Pathology of a tertiary care hospital in Mumbai, Maharashtra. Blood samples received for routine complete blood count in the hematology laboratory of 
the department were studied. Samples only from adult patients were included in the study. Data was recorded in a preformed proforma containing demographic details of patients along with hematological parameters, histograms and provisional clinical diagnosis. Histograms of these samples were obtained from a 3 part automated hematology analyzer, ERMA PCE 210.The hematological parameters were recorded from the automated hematology analyzer. Peripheral smears were prepared and stained with Romanowsky stains. Histograms obtained were evaluated in relation to the RBCs, WBCs and platelets with their peripheral blood smear (PBS) picture and with the cases with diagnosis of acute febrile illness. Sensitivity of hematology histogram in relation to the particular parameter was calculated by number of cases for that said parameter on histogram divided by number of cases on PBS and expressed as percentage.

Statistics: All the categorical and quantitative data was presented as frequency and proportion. Analysis of data was done using Microsoft Excel® 2010.

\section{Results}

i. Demographics of cases

A total of 200 consecutive samples were evaluated. 46 (23\%) samples were from 15-25 years age group followed by 41 (20.5\%) samples from 46-55 years age group. There was a single sample from age group 76-85 years. Out of 200 samples, 126 were from male patients. Demographics of the cases is presented in Table 1 and Fig.1.

ii. Distribution of cases based on histogram findings

Of the total 200 histograms, 26 histograms were normal. 30 histograms were having only WBC abnormality while 19 had RBC abnormality and 23 had platelet abnormality. 102 histograms had combined abnormality of RBCs, WBCs and platelets in varied combinations. Out of these 102, 43 had abnormality involving all three cell types, 30 had WBC and platelet abnormality, 23 had WBC and RBC abnormality while six had abnormality with RBCs and platelets. Distribution of cases based on histogram findings is presented in Fig.2

iii. Profile of findings of WBC histograms

Of the 200 cases, normal WBC histogram was seen with 74 cases. Neutrophilia (Fig 3) was seen with 90 cases, lymphocytosis in 35 cases while middle cell peak (Fig 4) was seen in one case. No case showed upper or lower discriminator. Profile of findings of WBC histograms is presented in Fig 5. iv. Sensitivity of WBC histogram with reference to PBS findings

Neutrophilia was seen in 111 cases on PBS and in 90 cases on histogram. Sensitivity of WBC histogram for neutrophilia was $81.08 \%$. Likewise sensitivity of WBC histogram for lymphocytosis was $77.8 \%$ while that for eosinophilia and blasts was 50\%. Sensitivity of WBC histogram with reference to PBS findings is presented in Table 2.

v. Profile of findings of RBC histograms

Of the total 200 cases, 109 cases had normal RBC histogram. 61 cases showed shift to left (microcytosis) (Fig 6), 16 showed shift to right (macrocytosis) while dimorphic (bimodal) RBC histogram (Fig 7) was seen with 14 cases. Profile of findings of RBC histograms is depicted in Fig. 8.

vi. Sensitivity of RBC histogram with reference to PBS findings

Microcytosis was seen with 75 cases on PBS and 61 cases on histogram. Sensitivity of RBC histogram in cases of microcytosis was $81.3 \%$. Similarly, sensitivity of $\mathrm{RBC}$ histogram in cases of macrocytosis was $80 \%$ while that in dimorphic (Bimodal) cases was 46.7\%. Sensitivity of RBC histogram with reference to PBS findings is presented in Table 3 .

vii. Profile of findings of platelet histograms

Of the total 200 cases, 98 cases showed normal platelet histogram. In 78 cases there was thrombocytopenia while two cases showed thrombocytosis on platelet histogram. Platelet histogram showed abnormality at upper discriminator (UD) in 22 cases (Fig 9). Profile of findings of platelet histogram is presented in Fig 10.

viii. Sensitivity of platelet histogram with reference to PBS findings

Thrombocytopenia was seen with 80 cases on PBS while platelet histogram showed thrombocytopenia in 78 cases. Sensitivity of platelet histogram in cases with thrombocytopenia was $97.5 \%$. Sensitivity of platelet histogram in cases of thrombocytosis was $50 \%$. Sensitivity of platelet histogram in cases of abnormality at upper discriminator due to cell fragments, microspherocytes or nucleated RBCs was $73.3 \%$. Sensitivity of platelet histogram with reference to PBS findings is presented in Table 4.

ix. Distribution of cases of Acute febrile illness

In the present study, acute febrile illness as diagnosis was mentioned for 82 cases. Of these, 32 patients were diagnosed with dengue followed by septicemia (11 cases) and urinary tract infection (9 cases). Pyogenic meningitis was diagnosed in two patients. Distribution 
of cases of acute febrile illness is presented in Fig 11.

x. Profile of parameters in cases of acute febrile illness

Out of total 82 cases with acute febrile illness, 61 cases showed thrombocytopenia (Fig 12). Leucopenia and leucocytosis were seen in 20 cases each while 37 cases showed atypical lymphocytes. Pancytopenia was seen in 08 cases. Profile of parameters in cases of acute febrile illness is presented in Table 5.

Table 1: Age wise distribution of cases.

\begin{tabular}{|c|c|c|}
\hline Age group (in years) & No. of Cases (N=200) & 23 \\
\hline $15-25$ & 46 & 14.5 \\
\hline $26-35$ & 29 & 18 \\
\hline $36-45$ & 36 & 20.5 \\
\hline $46-55$ & 41 & 15 \\
\hline $56-65$ & 30 & 8.5 \\
\hline $66-75$ & 17 & 0.5 \\
\hline $76-85$ & 1 & Percentage (\%) \\
\hline
\end{tabular}

Table 2: Sensitivity of WBC histogram with reference to PBS findings.

\begin{tabular}{|c|c|c|c|}
\hline Conditions & Number of cases on PBS & $\begin{array}{c}\text { Number of cases on } \\
\text { histogram }\end{array}$ & Sensitivity (\%) \\
\hline Neutrophilia & 111 & 90 & 81.08 \\
\hline Lymphocytosis & 42 & 35 & 77.8 \\
\hline Eosinophilia & 1 & 1 & 50 \\
Blasts & 1 & 74 & - \\
\hline Normal & 45 & & \\
\hline
\end{tabular}

Table 3: Sensitivity of RBC histogram with reference to PBS findings.

\begin{tabular}{|c|c|c|c|}
\hline Condition & Number of cases on PBS & $\begin{array}{c}\text { Number of cases on } \\
\text { histogram }\end{array}$ & Sensitivity (\%) \\
\hline Microcytosis & 75 & 61 & 81.3 \\
\hline Macrocytosis & 20 & 16 & 80 \\
\hline Dimorphic & 30 & 14 & 46.7 \\
\hline Normal & 75 & 109 & - \\
\hline
\end{tabular}

Table 4: Sensitivity of platelet histogram with reference to PBS findings.

\begin{tabular}{|c|c|c|c|}
\hline Condition & Number of cases on PBS & $\begin{array}{c}\text { Number of cases on } \\
\text { histogram }\end{array}$ & Sensitivity (\%) \\
\hline Thrombocytopenia & 80 & 78 & 97.5 \\
\hline Thrombocytosis & 04 & 02 & 50 \\
\hline Cell fragments & 11 & & \\
Microspherocytes & 09 & 22 & 73.3 \\
Nucleated RBCs & 10 & 98 & - \\
\hline Normal & 86 & & \\
\hline
\end{tabular}

Table 5: Profile of parameters in cases of acute febrile illness.

\begin{tabular}{|c|c|c|}
\hline Parameter & Number of cases & Percentage (\%) \\
\hline Leucopenia & 20 & 24.4 \\
\hline Leucocytosis & 20 & 74.4 \\
\hline Thrombocytopenia & 61 & 9.8 \\
\hline Pancytopenia & 08 & 45.1 \\
\hline Atypical lymphocytes & 37 & 24.4 \\
\hline
\end{tabular}




\section{Gender wise distribution of cases}

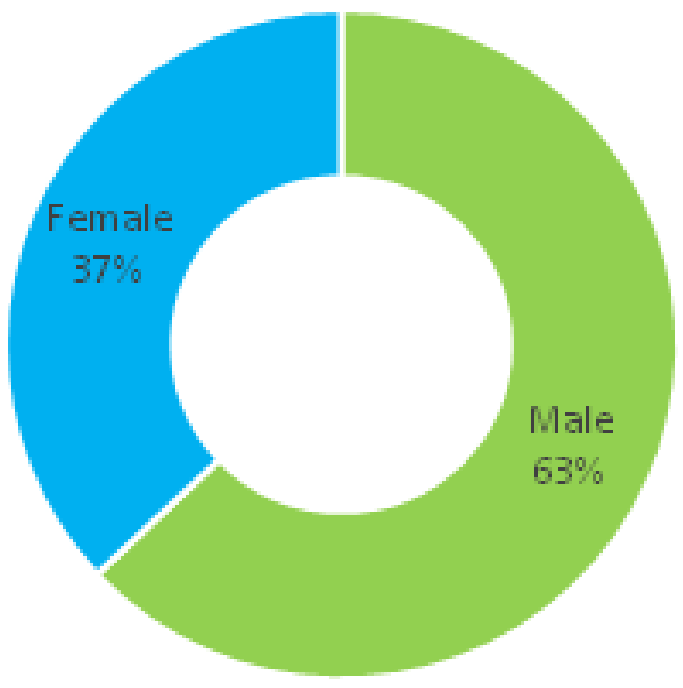

Fig. 1: Gender wise distribution of cases.

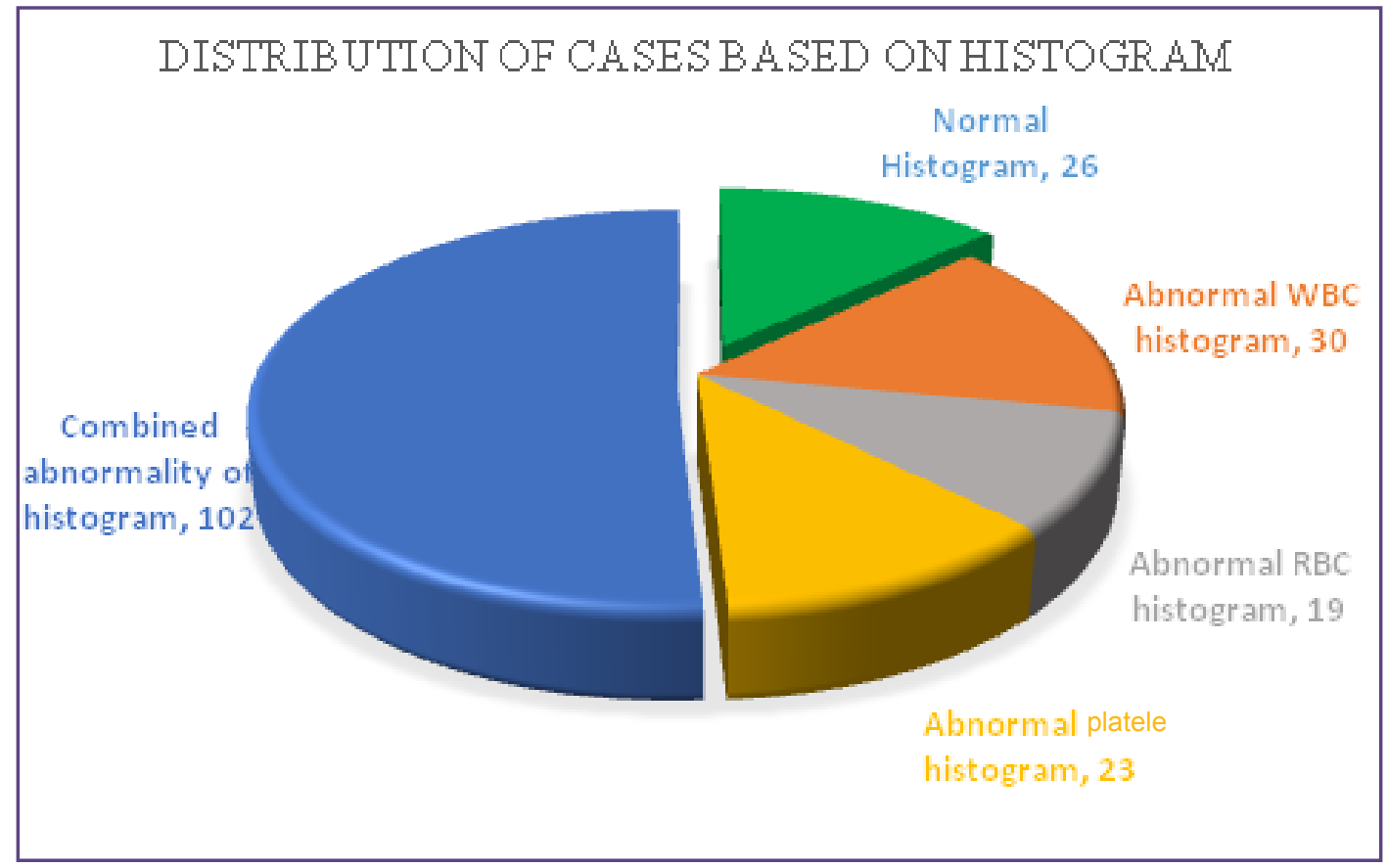

Fig. 2: Distribution of cases based on histogram findings. 


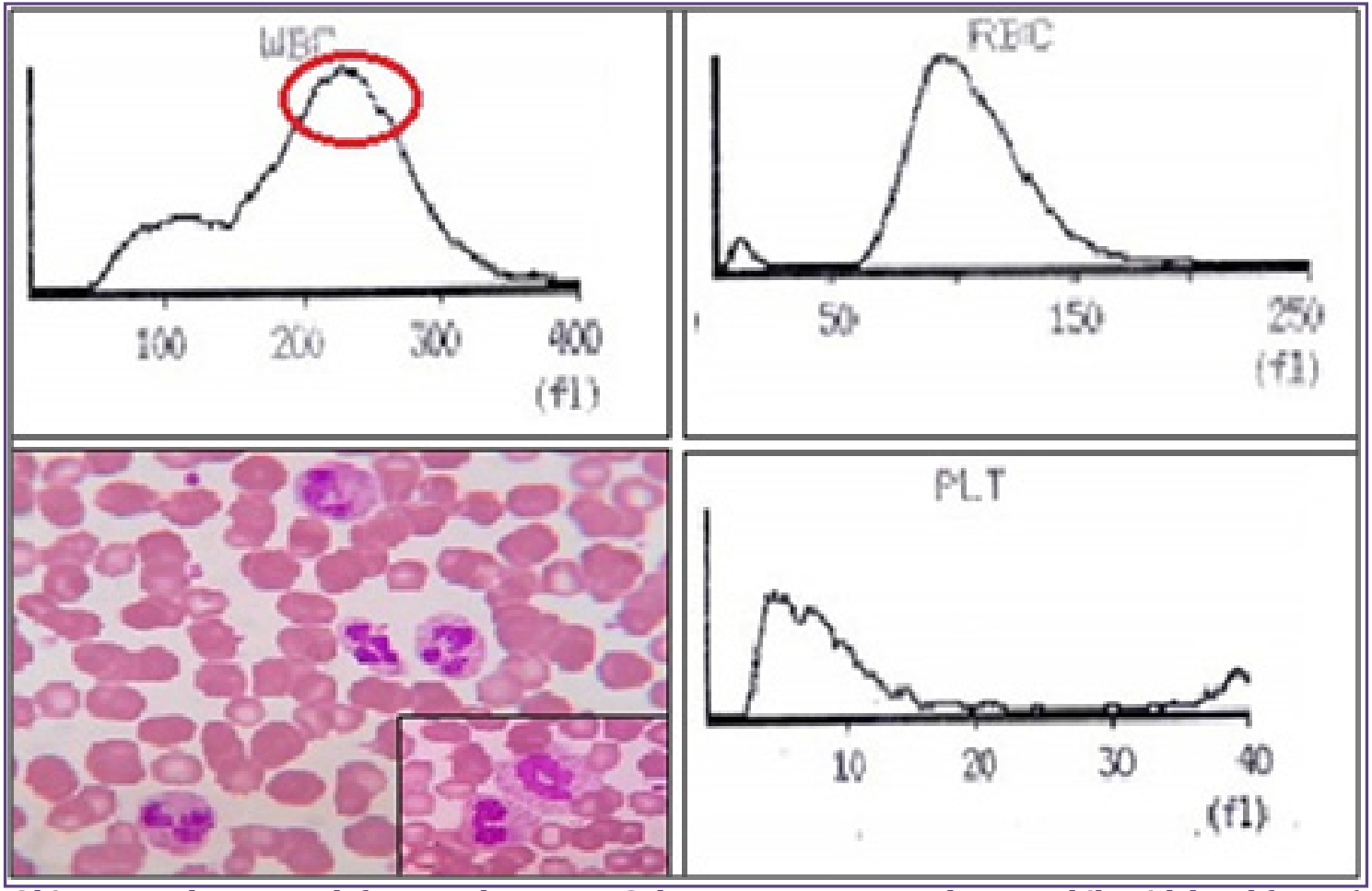

Fig. 3: WBC histogram shows a peak for granulocytes, PBS shows many segmented neutrophils with band forms (Inset) in a case of neutrophilia.
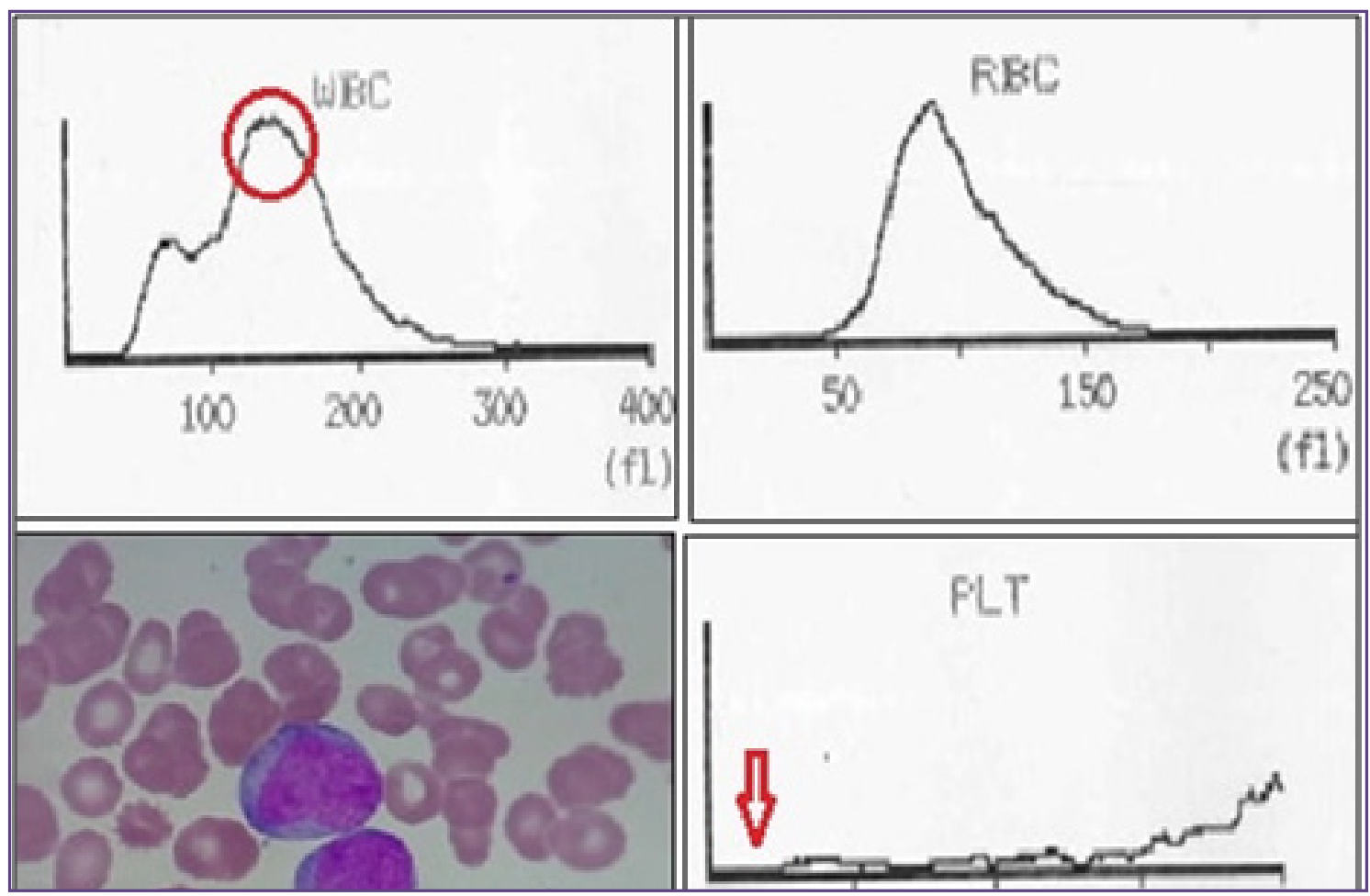

Fig. 4: WBC histogram shows a middle cell peak due to blasts .Platelet histogram shows thrombocytopenia. PBS shows blasts in a case of acute myeloid leukemia. 


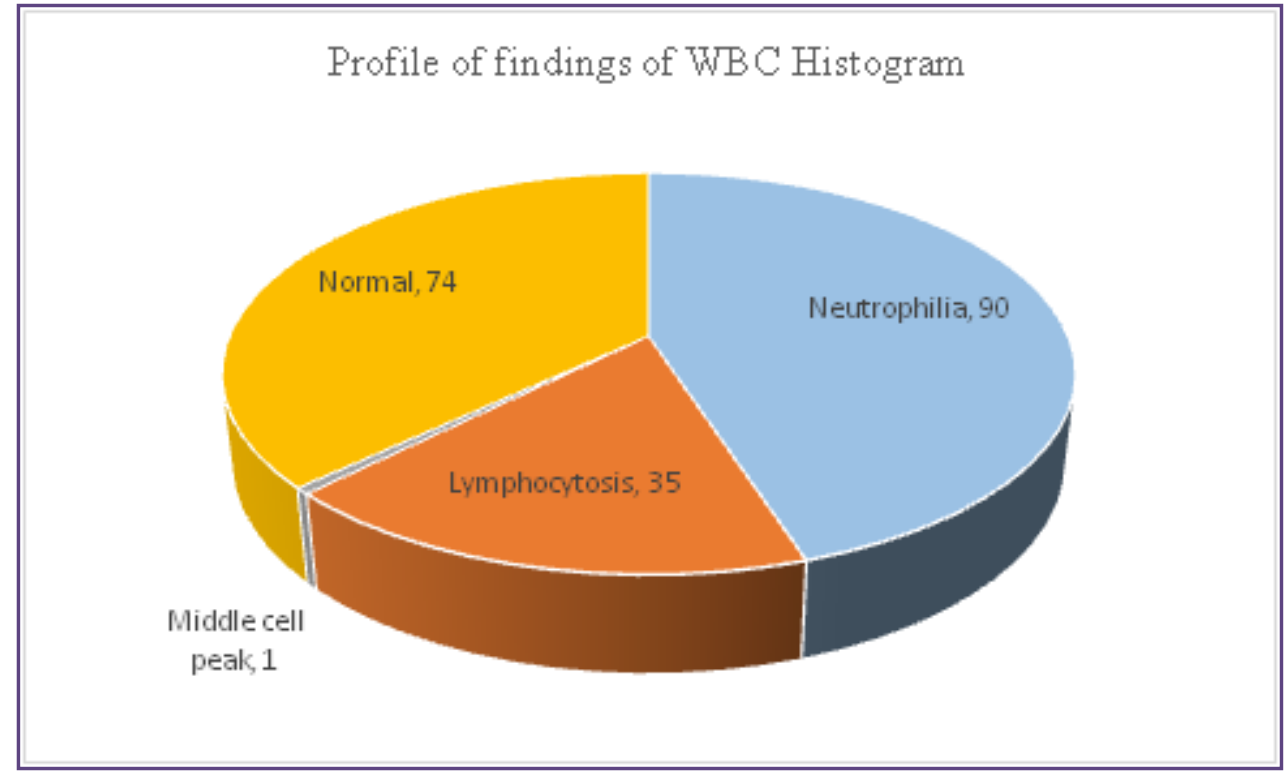

Fig. 5: Profile of findings of WBC histograms.

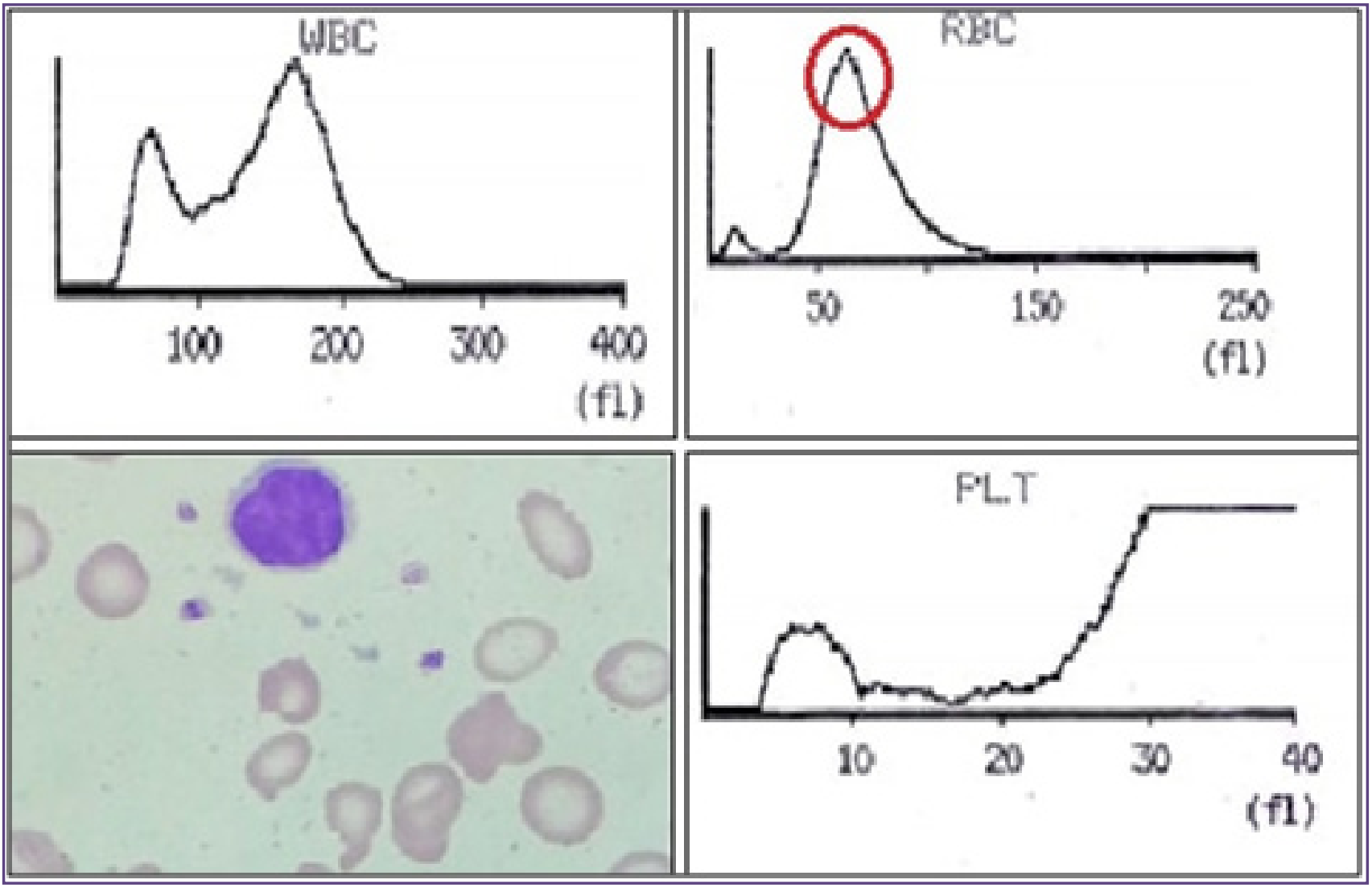

Fig. 6: RBC histogram shows shift to left due to microcytic RBCs. PBS shows microcytic, hypochromic RBCs in a case of iron deficiency anemia. 

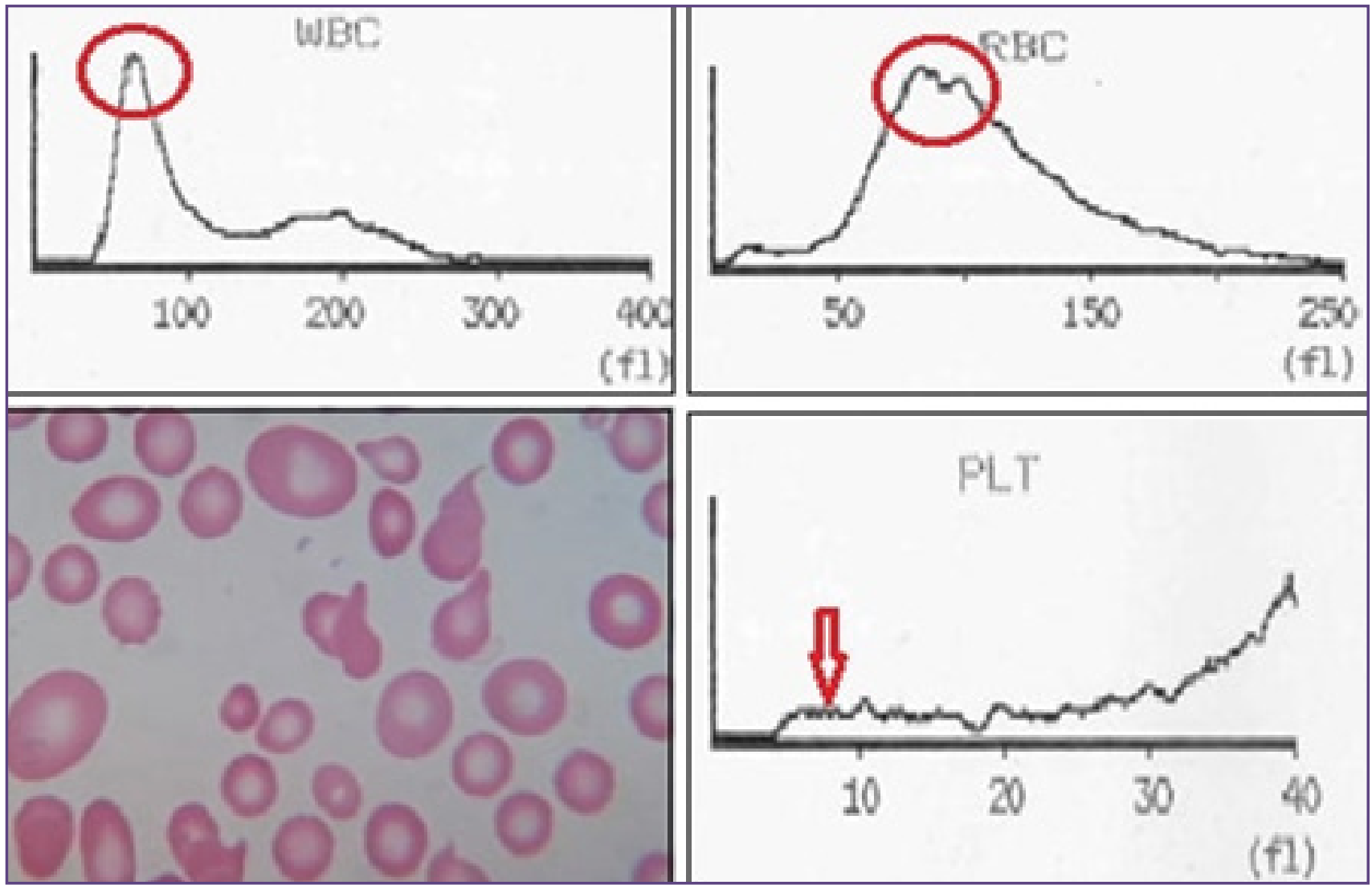

Fig. 7: RBC histogram shows bimodal peak, platelet histogram shows thrombocytopenia, WBC histogram shows peak for lymphocytes. PBS shows microcytes and macrovalocytes in a case of dimorphic anemia.

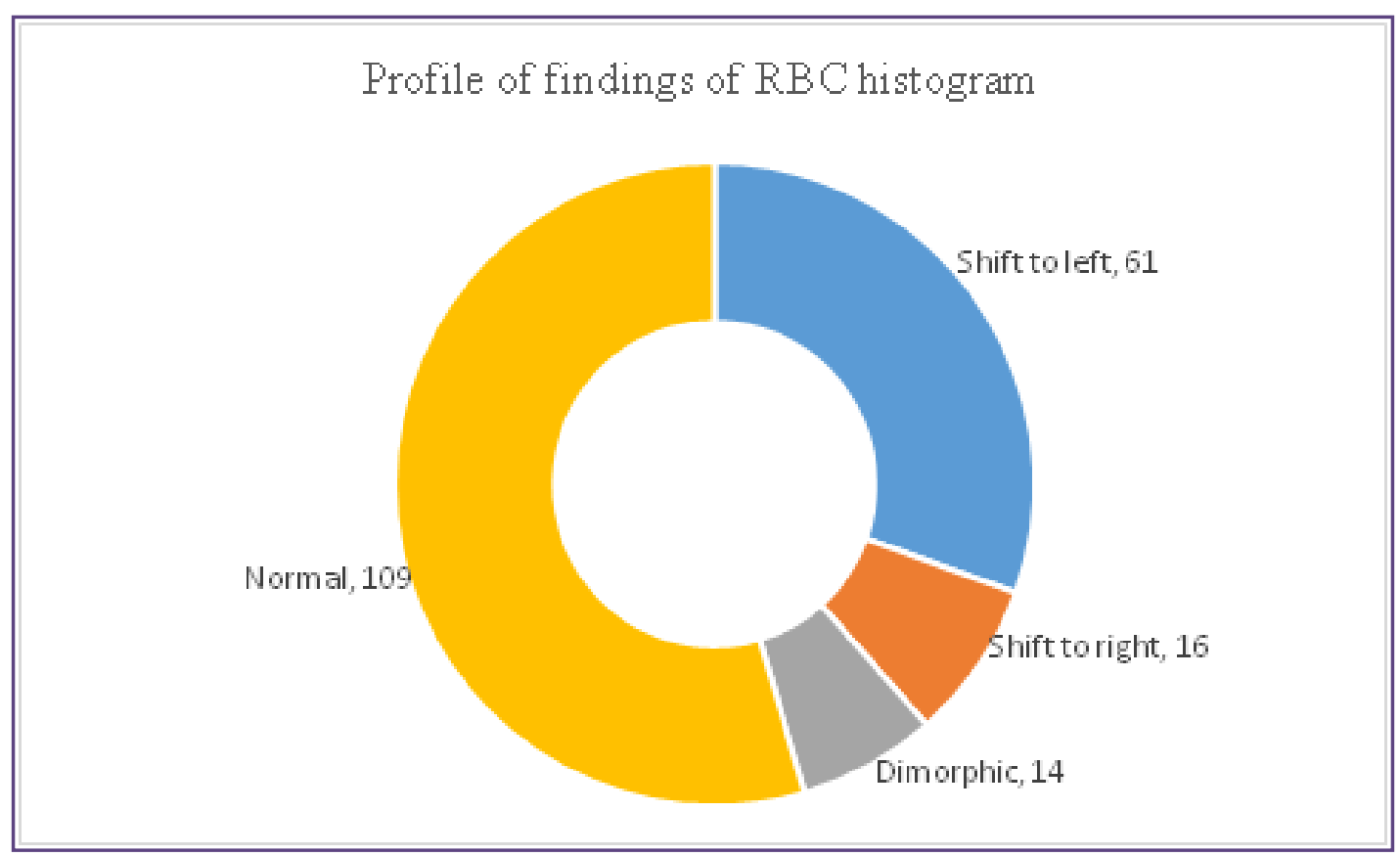

Fig. 8: Profile of findings of RBC histograms. 


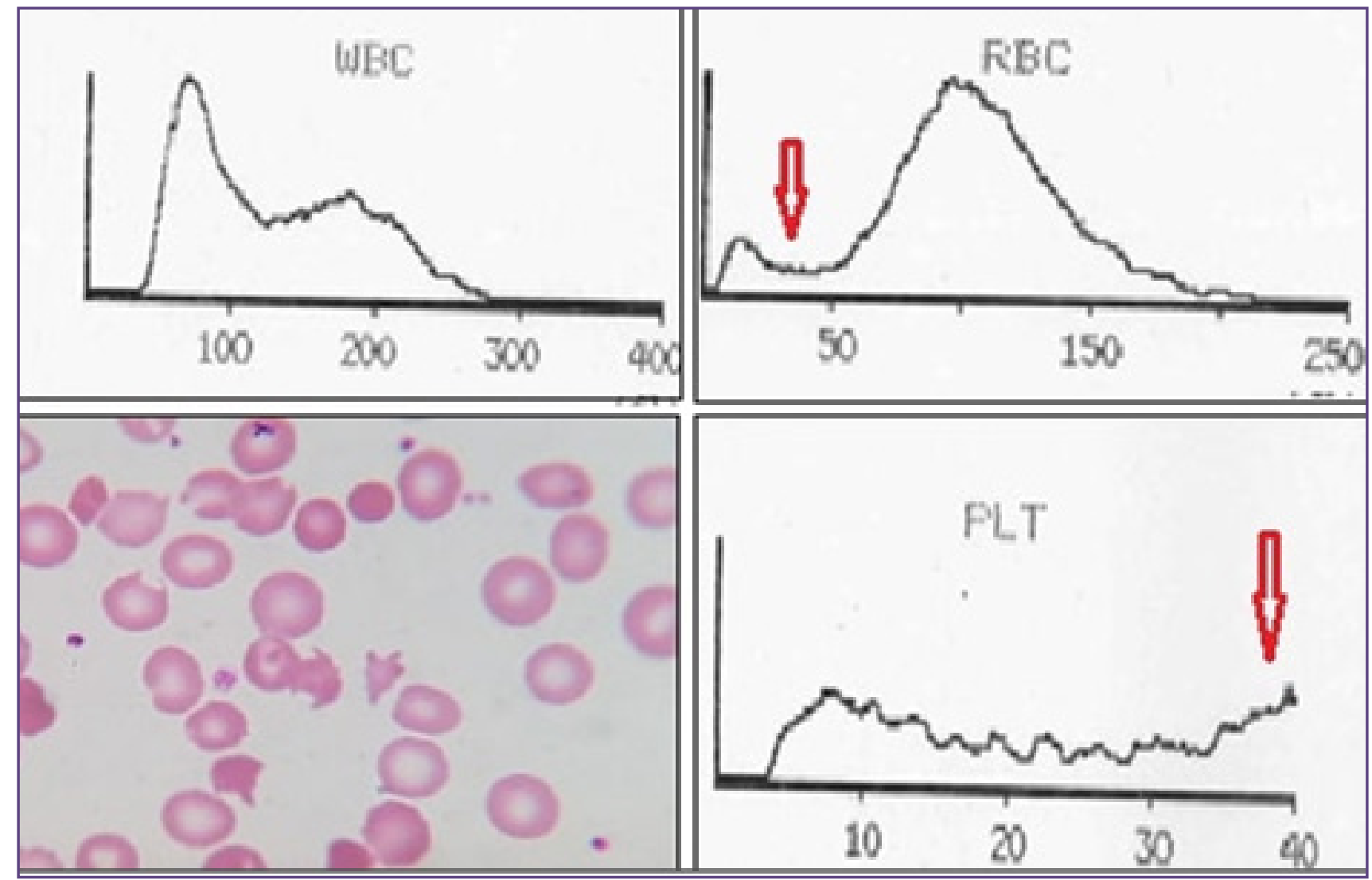

Fig. 9: Platelet histogram shows abnormality of upper discriminator and RBC histogram shows abnormality of lower discriminator.PBS shows schistocytes.

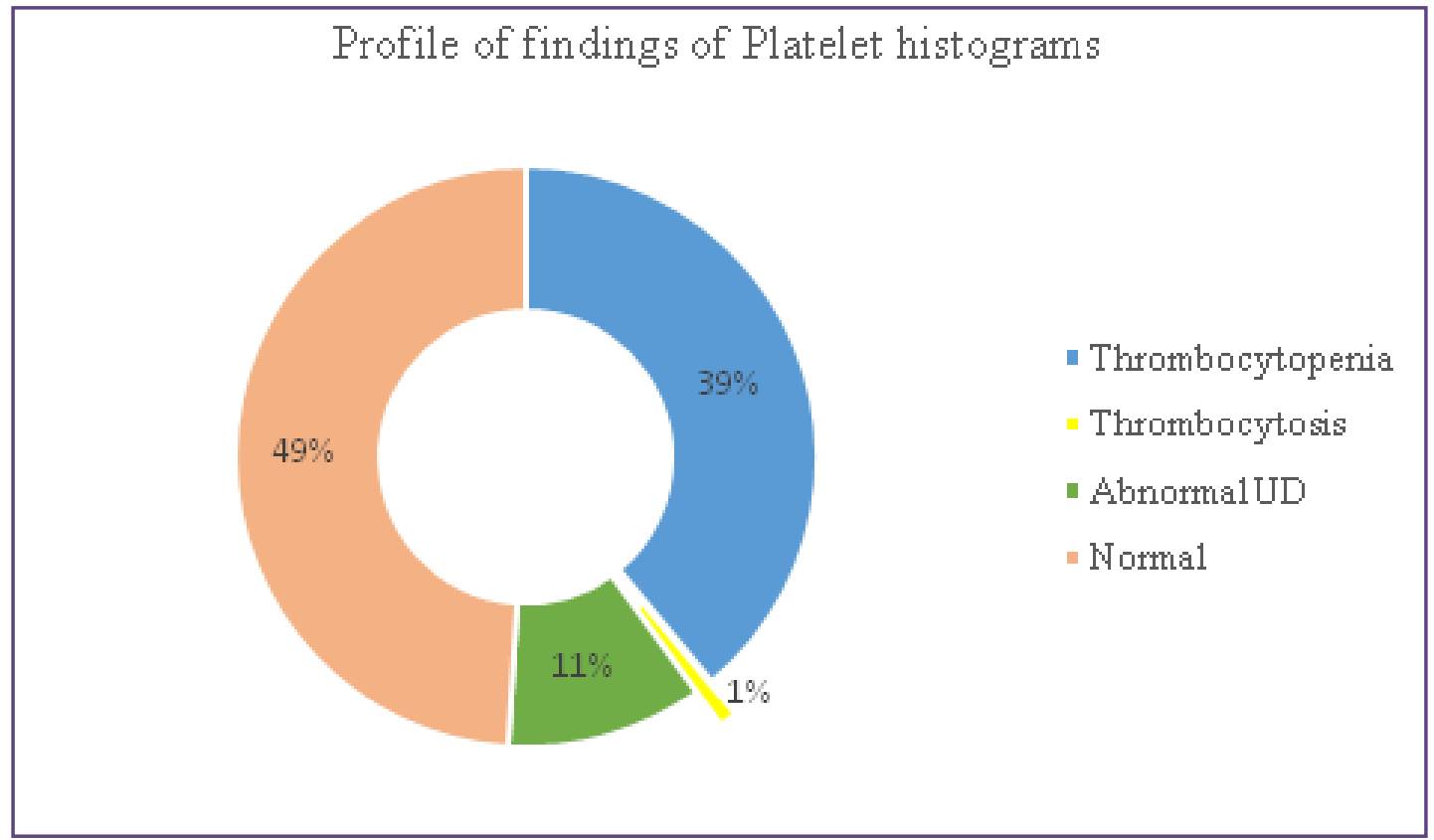

Fig. 10: Profile of findings of platelet histogram. 


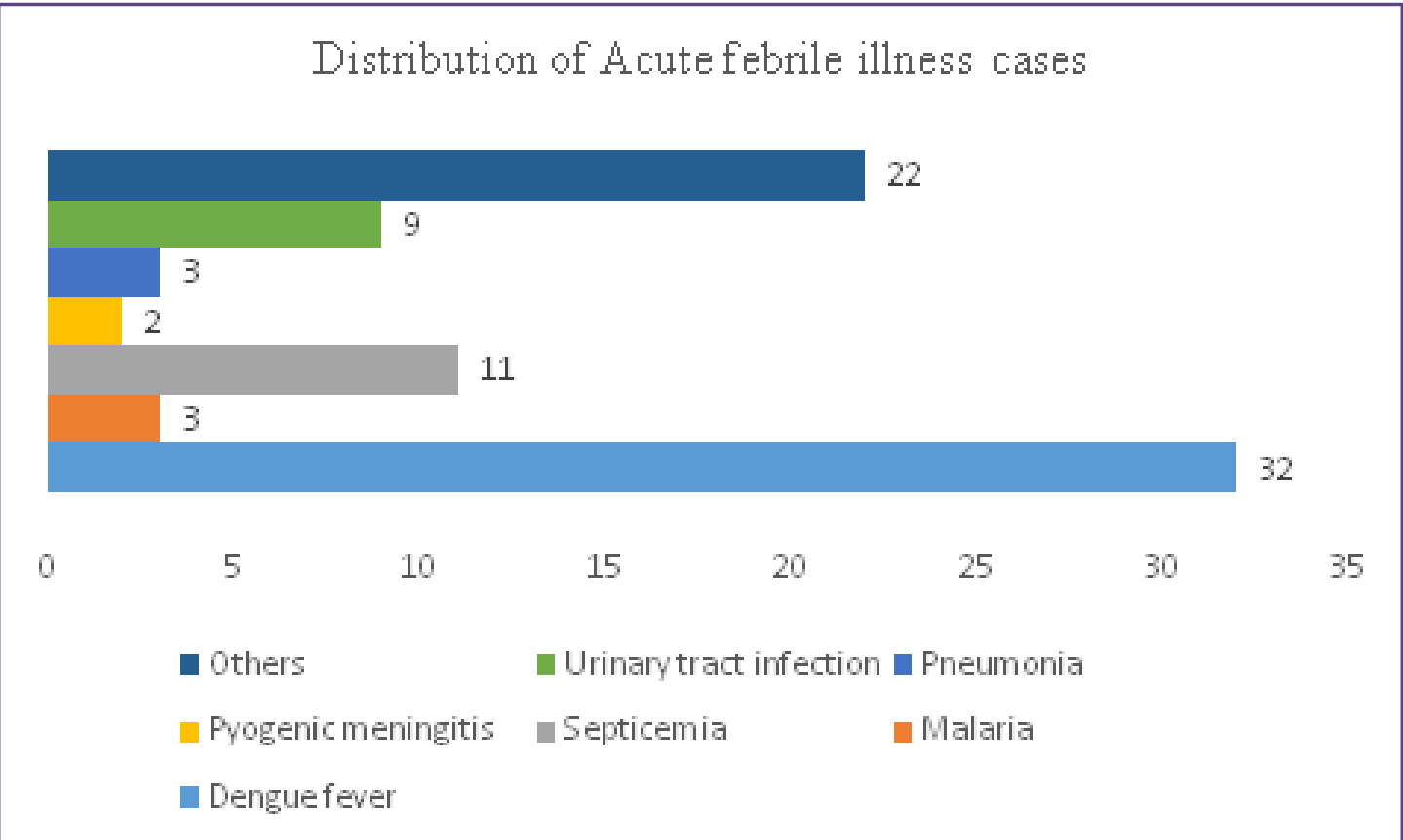

Fig. 11: Distribution of cases of acute febrile illness.

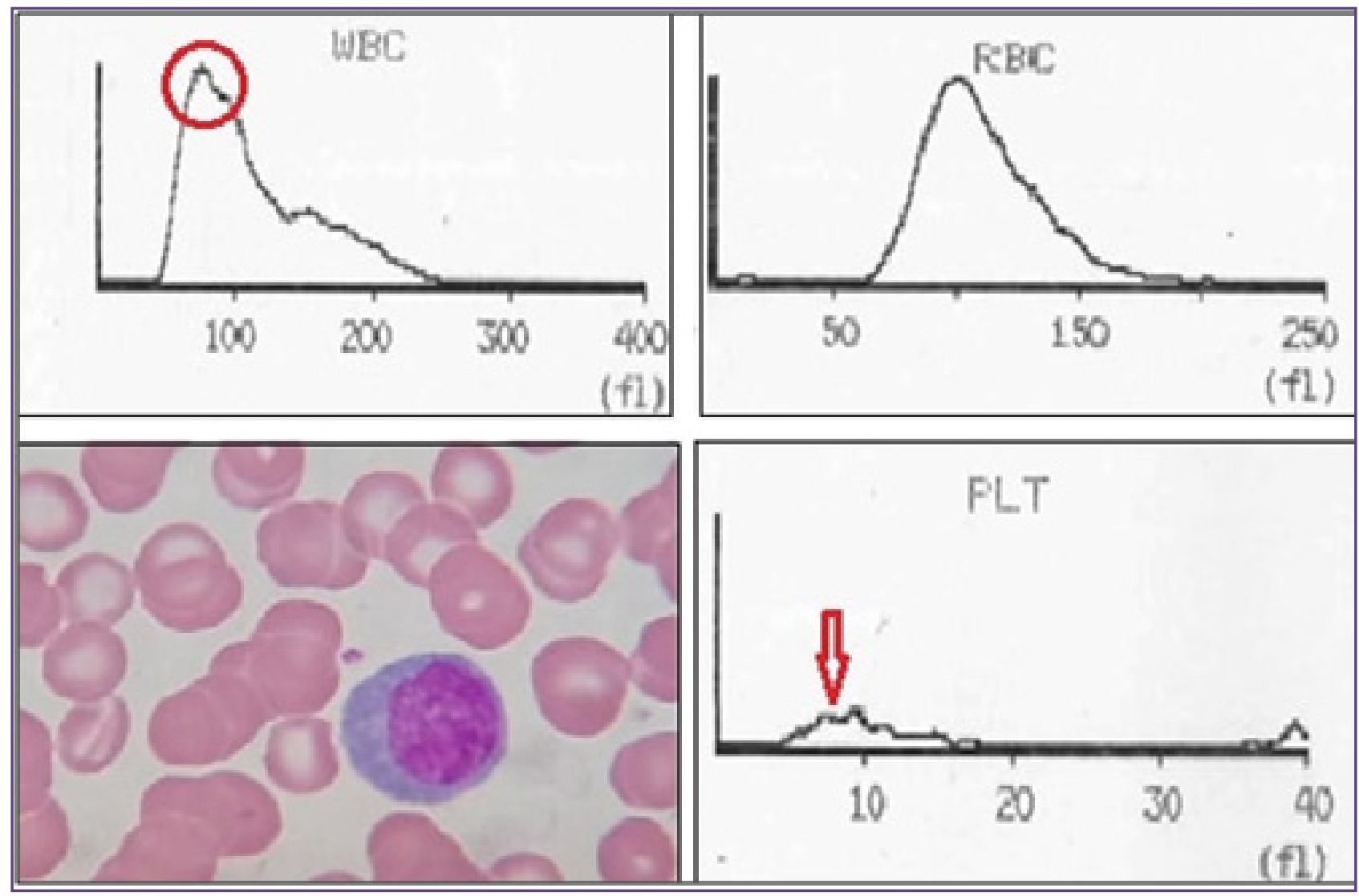

Fig. 12: WBC histogram shows peak for lymphocytes, Platelet histogram shows thrombocytopenia. PBS shows a plasmacytoid atypical lymphocyte in a case of dengue fever. 


\section{Discussion}

From reviewing the histograms, one can get a good idea of what to expect when actually evaluating the peripheral blood film. Unfortunately, most technologists have a limited understanding in correlating the graphic displays with the morphological findings. ${ }^{[2]}$

i. Demographics of the cases

A total of 200 consecutive samples were evaluated. $46(23 \%)$ samples were from $15-25$ years age group followed by $41(20.5 \%)$ samples from $46-55$ years age group. There was a single sample from age group 76-85 years. Out of 200 samples, 126 were from male patients.These findings are contrasting with those of Shrivastava et al. where maximum cases were from age group of 31 to 40 years followed by age group of 21 to 30 years and 686/1500 samples were of males. ${ }^{[3]}$ These differences in the findings can be attributed to the difference in the case pool.

ii. Distribution of cases based on histogram findings

Of the total 200 histograms, 26 histograms were normal. 30 histograms were having only WBC abnormality while 19 had RBC abnormality and 23 had platelet abnormalities. 102 histograms had combined abnormality of RBCs, WBCs and platelets in varied combinations. Out of these 102, 43 had abnormality involving all three cell types, 30 had WBC and platelet abnormality, 23 had WBC and RBC abnormality while six had abnormality with RBCs and platelets.

In spite of the extensive literature search, we did not come across any literature providing data regarding the comparatives of histograms of all three blood cell lineages. However, more than half of the histogram from the present study had combined abnormality of WBCs, RBCs and platelets. This points towards complex nature of disease processes in general.

iii. Profile of findings of WBC histograms

Of the 200 cases, normal WBC histogram was seen with 74 cases. Neutrophilia was seen with 90 cases, lymphocytosis in 35 cases while middle cell peak was seen in one case. No case showed upper or lower discriminator.

iv. Sensitivity of WBC histogram with reference to PBS findings

Neutrophilia was seen in 111 cases on PBS and in 90 cases on histogram. Sensitivity of WBC histogram for neutrophilia was $81.08 \%$. Likewise sensitivity of WBC histogram for lymphocytosis was $77.8 \%$ while that for eosinophilia and blasts was 50\%.

These findings are in in concordance with the study of Petani S et al. which shows $85.4 \%$ sensitivity for immature granulocytes or left shift. ${ }^{[4]}$ v. Profile of findings of RBC histograms

Of the total 200 cases, 109 cases had normal RBC hstogram. 61 cases showed shift to left , 16 showed shift to right while dimorphic (bimodal) RBC histogram was seen with 14 cases.

These findings are comparable with the findings of Shrivastava et al., Sandhya et al., Chavda et al. and Rao BSS et al.

\begin{tabular}{|l|l|l|l|l|l|}
\hline Histogram & $\begin{array}{l}\text { Shrivastava } \\
\text { et al. }{ }^{[3]}\end{array}$ & $\begin{array}{l}\text { Sandhya } \\
\text { et al. }\end{array}$ & $\begin{array}{l}\text { Chavda } \\
\text { et al. } .^{[6]}\end{array}$ & $\begin{array}{l}\text { Rao BSS } \\
\text { et al. }{ }^{7]}\end{array}$ & $\begin{array}{l}\text { Present } \\
\text { study }\end{array}$ \\
\hline Normal & $18 \%$ & $15 \%$ & $19 \%$ & $17.70 \%$ & $54.5 \%$ \\
\hline Left Shift & $29 \%$ & $30 \%$ & $27 \%$ & $29 \%$ & $30.5 \%$ \\
\hline Right Shift & $06 \%$ & $06 \%$ & $07 \%$ & $05.45 \%$ & $08 \%$ \\
\hline Bimodal & $05 \%$ & $04 \%$ & $03 \%$ & $7.27 \%$ & $07 \%$ \\
\hline
\end{tabular}

vi Sensitivity of RBC histogram with reference to PBS findings

Microcytosis was seen with 75 cases on PBS and 61 cases on histogram. Sensitivity of RBC histogram in cases of microcytosis was $81.3 \%$. Similarly, sensitivity of RBC histogram in cases of macrocytosis was $80 \%$ while that in dimorphic cases was $46.7 \%$.

Higher sensitivity points towards the fact that RBC histogram is a relatively reliable method to detect microcytosis and macrocytosis. However, dimorphic (bimodal) picture of RBCs can be detected in $50 \%$ of cases using RBC histogram only.

vii. Profile of findings of platelet histograms

Of the total 200 cases, 98 cases showed normal platelet histogram. 78 cases showed thrombocytopenia while two cases showed thrombocytosis on platelet histogram. Platelet histogram showed abnormality at upper discriminator (UD) in 22 cases.

viii. Sensitivity of platelet histogram with reference to PBS findings

Thrombocytopenia was seen with 80 cases on PBS while platelet histogram showed thrombocytopenia in 78 cases. Sensitivity of platelet histogram in cases with thrombocytopenia was $97.5 \%$. Sensitivity of platelet histogram in cases of thrombocytosis was $50 \%$. Sensitivity of platelet histogram in cases of abnormality at upper discriminator (UD) due to cell fragments, microspherocytes or nucleated RBCs was $73.3 \%$, this is comparable to study by Gupta et al. which had $100 \%$ sensitivity of platelet histograms in cases with UD abnormality. ${ }^{[8]}$

Higher sensitivity of platelet histogram for thrombocytopenia suggests that platelet histogram is as effective as PBS as far as diagnosis of 
thrombocytopenia is considered. However, platelet histogram is only half good as compared to PBS to detect thrombocytosis.

ix. Distribution of cases of Acute febrile illness

In the present study, acute febrile illness as diagnosis was mentioned for 82 cases. Of these, 32 patients were diagnosed with dengue followed by septicemia (11 cases) and urinary tract infection (9 cases). Pyogenic meningitis was diagnosed in two patients.

These findings are in line with findings those of Muthaiah et al. where dengue was the most common (28\%) cause of the acute febrile illness. ${ }^{[9]}$

x. Profile of parameters in cases of acute febrile illness

Out of total 82 cases with acute febrile illness, 61 cases showed thrombocytopenia. Leucopenia and leucocytosis were seen in 20 cases each while 37 cases showed atypical lymphocytes. Pancytopenia was seen in 08 cases.In 29 cases $(47.5 \%)$ of acute febrile illness with thrombocytopenia the cause was dengue fever. This is comparable with the study of Raikar $\mathrm{S}$ et al. in which viremia was the commonest cause of thrombocytopenia including dengue, seen in 52\% cases. $^{[10]}$

\section{Conclusion}

Histograms provided by the automated hematology analyzers are of great diagnostic and morphologic importance. They alert us, if a peripheral blood smear preparation and examination is required or not, especially in centres with high sample load. From reviewing the histograms, we can get a good idea of what to expect when actually evaluating the peripheral blood smear.

Because graphics can show data in ways that are more meaningful and quickly understood, the histogram is a very powerful tool in morphological analysis of red blood cells, white blood cells and platelets. It enables one to visualize, analyse, and interpret empirical data that displays morphological changes graphically as points, peaks or valleys, or as a line of frequency curve. Visual scanning of the histogram gives a good initial sense of the range, size, shape and other salient features of the cell morphology.

Histograms are not meant to replace traditional manual review of peripheral smears. Rather, they should help to select those peripheral smears which require manual review. Histograms obtained from automated analyzers, should be complementary to hematological parameters and peripheral blood smear examination.

\section{References}

1. Bains BJ. Performing a blood count In: Blood Cells - A Practical Guide. 4th ed. London: Blackwell Science; 2006.

2. Sullivan E. Hematology analyzer: From workhorse to thoroughbred. Lab Med. 2006; 37:273-278.

3. Shrivastav A, Shah N, Goyal S, Shah CK. RBC histogram: Utility in diagnosis of various anemia. International Journal of Clinical and Diagnostic Pathology. 2019; 2(1): 14-17.

4. Petani S, Topic E, Turcic G, Däschner M. Clinical Evaluation of the Cell-Dyn ${ }^{\circledR}$ 1700CS Blood Counter. Clinical Chemistry. June 1997; 43 (6): 1085-1088.

5. Sandhya I, Muhasin TP. Study of RBC Histogram in various anemias. Journal of Evolution of Medical and Dental sciences. 2014; 3(74):15521-34.

6. Chavda J, Goswami P, Goswami A. RBC histogram as diagnostic tool in anemias. IOSR Journal of Dental and Medical Sciences. 2015; 14(10):19-22.

7. Rao BSS, Vissa S, Rao NM, Grandhi B, Muramreddy V, Sirasala P. RBC Histogram as Supplementary Diagnostic Tool with Peripheral Smear Examination in Evaluating Anaemia. Annals of Pathology and Laboratory Medicine. 2017; 4(6):A668-672

8. Gupta A, Gupta P, Bhaghat VM. Interpretation of histograms and its correlation with peripheral smear findings. J. Evolution Med. Dent. Sci. 2017;6(60):4417-4420,

9. Muthaiah B, Thippeswamy T, Kondareddy S, Chikkegowda P. Study of Aetiology and Outcome in Acute Febrile Illness Patients with Multiple Organ Dysfunction Syndrome. J Clin Diagn Res. 2016; 10(8):OC16-OC18.

10. Raikar S, Kamdar P, Dabhi A. Clinical and Laboratory Evaluation of Patients with Fever with Thrombocytopenia Indian Journal of Clinical Practice. 2013;24 (4): 360-363.

*Corresponding author:

Dr. Tejaswini Waghmare, Assistant Professor, Department of Pathology, 1st Floor, Dean Office Building, Seth G.S. Medical College and KEM Hospital, Parel, Mumbai. 400012

Phone: +91 9769912040

Email: tejaswinipanad@gmail.com

Financial or other Competing Interests: None. 